\section{PRIME-BOOST VACCINATION FOR THE TREATMENT OF TRIPLE NEGATIVE BREAST CANCER}

Seyedeh-Raheleh Niavarani*, Christine Lawson, Lee-Hwa Tai. Sherbrooke University, Sherbrooke, Canada

Background Triple negative breast cancer (TNBC) is currently only treated with surgery and chemotherapy. ${ }^{1}$ TNBC has higher rates of genetic mutations and contains more tumor infiltrating lymphocytes. ${ }^{2}$ These characteristics provide a strong rationale to use novel immunotherapies such as immunogenic autologous tumor cell vaccines to therapeutically target TNBC. We have demonstrated that an infected cell vaccine (ICV) that is made from irradiated and oncolytic virus tumor infected cells induces beneficial innate and adaptive immune responses in a syngeneic mouse model of TNBC. Moreover, the efficacy of ICV is improved when combined with checkpoint blockade (anti-PD-1). ${ }^{3}$ Our goal is to further improve ICV by applying a prime-boost cancer vaccination strategy to further enhance anti-tumor immune responses in preclinical and translational studies. $^{4} 5$

Methods We will choose the best 'prime vaccine' based on the immunogenicity of TNBC cell lines after treatment with immunomodulators such as chemotherapeutic agents, irradiation, tolllike receptor agonists and anti-viral vaccines. We will measure the release of damage-associated molecular patterns (DAMPs), which act as danger signals to initiate tumor-targeted immune responses, ${ }^{6}$ after the treatment of TNBC cell lines. We will test the polarization of human monocytes when co-cultured with conditioned media (CM) from treated TNBC cells. We will also analyze the migration of human immune cells (CD56+NK cells and $\mathrm{CD} 8+\mathrm{T}$ cells) toward the $\mathrm{CM}$ of treated human TNBC cells. Furthermore, we will evaluate the maturation markers on CD11C+ dendritic (DC) cells differentiated from mouse bone marrow cells when co-cultured with the cell lysate of the mouse TNBC cell line treated with 'prime vaccine' candidates. For in vivo studies, we will test our best prime vaccine followed by the ICV as a boost vaccine in our BALB/c-4T1 mouse model. We will analyze the cytotoxicity of $\mathrm{T}$ lymphocytes and the secretion of cytokines, and overall survival.

Results From measuring DAMP levels and analyzing immune functions, our preliminary results suggest that oxaliplatin and the seasonal influenza vaccine are the best candidates as strong 'prime vaccine' candidates compared to other treatments. DCs differentiated from isolated bone marrow cells exhibited a higher percentage of markers of maturation when co-cutured ex vivo with cell lysate of 4T1 cells were treated with oxaliplatin compared to control groups. In vivo studies in the BALB/c-4T1 model have begun to test the best prime-boost vaccine combinations.

Conclusions These results demonstrate the therapeutic potential of oncolytic virus-based immunogenic tumor vaccines could be improved by applying the 'prime-boost' cancer vaccination approach to treat TNBC.

Ethics Approval 'The study was approved by the CRCHUS Human Ethics Board, approval number 2018-2414.'

Consent 'Written informed consent was obtained from the patient for publication of this abstract and any accompanying images. A copy of the written consent is available for review by the Editor of this journal.'

\section{REFERENCES}

1. Yadav BS. Systemic treatment strategies for triple-negative breast cancer. World J Clin Oncol 2014;5(2):125.

2. García-Teijido P, Cabal ML, Fernández IP, Pérez YF. Tumor-infiltrating lymphocytes in triple negative breast cancer: the future of immune targeting. Clinical Medicine Insights: Oncology 2016
3. Niavarani SR, Lawson C, Boudaud M, Simard C, Tai LH. Oncolytic vesicular stomatitis virus-based cellular vaccine improves triple-negative breast cancer outcome by enhancing natural killer and CD8 + T-cell functionality. J Immunother Cancer 2020.

4. Le DT, et al. Safety and survival with GVAX pancreas prime and listeria monocytogenes-expressing mesothelin (CRS-207) boost vaccines for metastatic pancreatic cancer. J Clin Oncol 2015.

5. Pol JG, et al. Preclinical evaluation of a MAGE-A3 vaccination utilizing the oncolytic maraba virus currently in first-in-human trials. Oncoimmunology 2019 Jan;8 (1):e1512329.

6. Vandenberk L, Belmans J, Van Woensel M, Riva M, Van Gool SW. Exploiting the immunogenic potential of cancer cells for improved dendritic cell vaccines. Frontiers in Immunology 2016.

http://dx.doi.org/10.1136/jitc-2020-SITC2020.0848

\section{OPTIMIZATION OF A GM3-CONTAINING LIPOSOMAL VACCINE THAT DELIVERS ANTIGEN TO CD169+ SPLENIC MACROPHAGES}

${ }^{1}$ Maarten Nijen Twilhaar*, ${ }^{2}$ Lucas Czentner Colomo, 'Joanna Grabowska, ${ }^{1}$ Alsya Affandi, ${ }^{1}$ Katarina Olesek, ${ }^{1}$ Hakan Kalay, ${ }^{2}$ Rene van Nostrum, ${ }^{3}$ Paul Crocker, ${ }^{1}$ Yvette van Kooyk, ${ }^{2}$ Gert Storm, 'Joke den Haan. 'Amsterdam UMC, Amsterdam, Netherlands; ${ }^{2}$ Utrecht University, Utrecht, Netherlands; ${ }^{3}$ University of Dundee, Dundee, UK

Background Although promising developments in cancer vaccination have been made, therapeutic effectiveness is often insufficient. Liposomal vaccine effectiveness could be enhanced by antigen encapsulation and incorporation of molecules that actively target to antigen presenting cells to enhance $\mathrm{T}$ cell activation. CD169expressing splenic macrophages are located in the marginal zone and efficiently capture particulate antigens such as viruses and exosomes from the blood circulation. Upon antigen capture CD169+ macrophages transfer antigen to cross-presenting dendritic cells that are responsible for the activation of $\mathrm{CD} 8+\mathrm{T}$ cells.

Methods Here we prepared liposomes that contain a physiological ligand for CD169, the ganglioside GM3, to facilitate uptake by CD169+ macrophages. We assessed how various amounts of targeting molecule GM3, decoration with PEG and liposomal size affected binding and uptake by CD169+ macrophages in vitro and in vivo. In addition, we evaluated the stability of liposomal preparations in plasma. As a proof of concept, we prepared GM3-liposomes with a long ovalbumin peptide and tested the capacity of these liposomes to induce $\mathrm{CD} 8+$ and $\mathrm{CD} 4+\mathrm{T}$ cell activation and compared it to control liposomes and soluble peptide.

Results These data indicate that targeting of splenic CD169 macrophages can be optimized by careful selection of constituents of the liposomal delivery vehicle. Moreover, optimized GM3-mediated liposomal targeting to CD169 macrophages results in potent immune responses.

Conclusions GM3-mediated liposomal targeting to CD169 macrophages presents as a promising strategy for cancer vaccines

Ethics Approval All animal experiments were approved by the local animal welfare body.

http://dx.doi.org/10.1136/jitc-2020-SITC2020.0849

\section{IMMUNOLOGICAL CONSIDERATIONS FOR DEVELOPING OPTIMAL WHOLE TUMOR CELL-DERIVED CANCER VACCINES}

Flavio Salazar Onfray*. Universidad de Chile, Santiago, VT, Chile

Background Immunotherapies based on checkpoint blockers (ICB), targeting inhibitory immune pathways such as cytotoxic T 